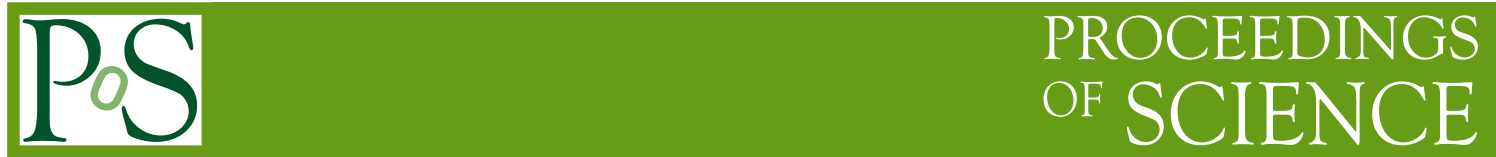

\title{
Studies on the antikaon-nucleon interaction with the KLOE Drift Chamber
}

\section{Oton Vázquez Doce*}

Excellence Cluster Universe (TUM)

E-mail: oton.vazquez@universe-cluster.de

The study of the $\mathrm{K}^{-}$hadronic interactions inside the drift chamber of KLOE constitute a first step towards the preparation of the AMADEUS experiment at DAFNE, the $\mathrm{e}^{+} \mathrm{e}^{-}$collider of the Frascati National Laboratories (Italy) of INFN.

The absorption of antikaons in light nuclei are studied, thanks to the KLOE detector, with almost full acceptance. This kind of studies provide information concerning the modification of the $\bar{K} N$ potential in the nuclear medium.

We report here the analysis of the $\Lambda p$ pairs correlations produced in the absorption of $K^{-}$inside the volume of the KLOE drift chamber, filled mainly by ${ }^{4} \mathrm{He}$.

International Winter Meeting on Nuclear Physics,

21-25 January 2013

Bormio, Italy

\footnotetext{
${ }^{*}$ Speaker.
} 


\section{Introduction}

The investigation of the absorptions of $K^{-}$inside the KLOE drift chamber was motivated by the prediction of the formation of deeply bound kaonic nuclear states [1]. This kind of kaonic clusters would be formed when a $K^{-}$is attached inside a nucleus, and its binding energy and width could be determined studying their decay into baryons and nucleons. In particular the dibarionic state $K^{-} p p$ is expected to decay into $\Lambda p$.

The modification in the nuclear medium of the properties and interactions of the antikaons is being sistematically studied from the theoretical and experimental point of view.

Different predictions for the strenght of the antikaon-nucleon potential exist, leading to different values for the binding energies of the $K^{-}$nuclei. Recently, the publication of the results from the SIDDHARTA experiment [2], providing the scattering length for $K^{-} p$ with unprecedent accuracy, allowed to have more precise predictions on the value of the antikaon-nucleon potential in the nuclear environment.

From the experimental point of view, two main approaches have been used for studying the $K^{-} p p$ cluster: $p-p$ and heavy ion collisions [3] [4], and low momentum or stopped $K^{-}$interactions in light nuclei. Among the second, data has been published by the FINUDA [5] and KEK-PS E549 collaborations [6]. The interpretation of both results is far from being conclusive, and it requires an accurate description of the single and multi-nucleon absoption processes that a $K^{-}$would undergo when interacting with light nuclei. These processes are poorly known from the experimental point of view, and the branching ratios have been extracted using only 1000 events from a bubble chamber experiment [7].

The correct interpretation of the experimental results, including as well the old emulsion and helium bubble chamber experiments that have presented analog invariant mass spectra and correlations of $\Lambda N$ (see, for example [8]), requires a precise knowledge of the shape and characteristics of the single and multi-nucleon absoption processes, in order to decouple this type of background from an expected signal due to the formation of a bound state. The success in the detection of the kaonic clusters will be based on this knowledge, as well as on the width and binding energies inferred to the bound states by the antikaon-nucleon potential in the nuclear environment.

In this paper we present the analysis of the $\Lambda p$ events coming from the $K^{-}$interactions in the KLOE drift chamber volume. It will be shown how it was possible as well to obtain a exclusive measurement of $\Lambda p \pi^{-}$events, that are produced mainly in the single nucleon absorption process.

\section{Events selection in KLOE}

A previous study using the standard KLOE Monte Carlo simulation (GEANT), was done in order to calculate the expected number of stopped $\mathrm{K}^{-}$inside the drift chamber (DC). The fraction of $\mathrm{K}^{-}$that stops in the gas volume was estimated to be $0.1 \%$, which opened the possibility for studies of the $K^{-}$interactions at rest with the available 2004-2005 KLOE data. The data presented here confirms that the KLOE detector [9] is a good environment for this kind of research, the precision achieved being better than any other recently published data, although the lack of a real target is translated in a (expected) low number of events. 


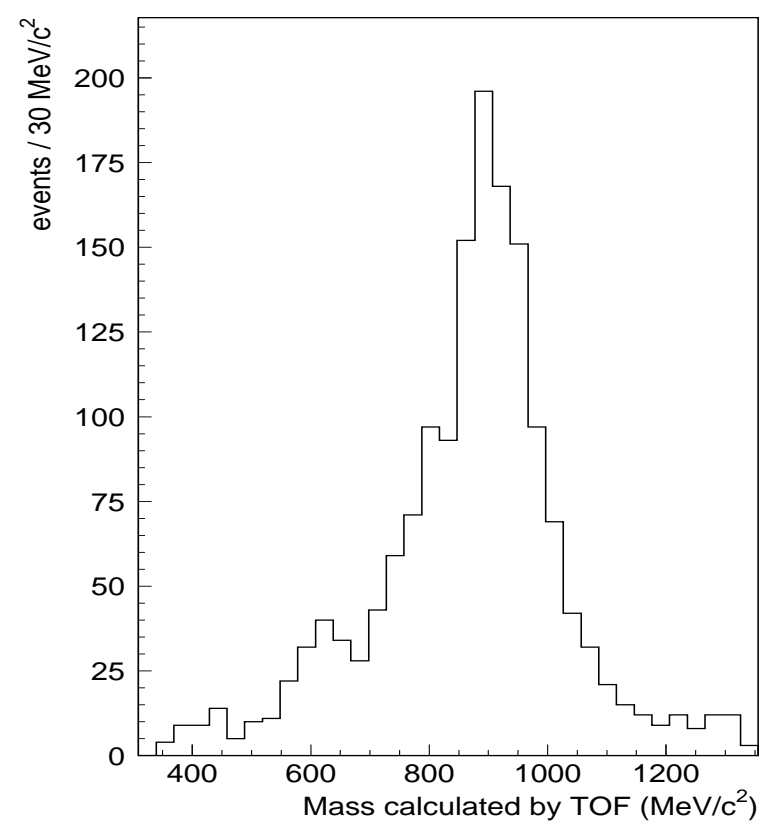

Figure 1: Mass calculated by time of flight for the proton tracks with an associated cluster in the electromagnetic calorimeter.

The sample of events used comprehends a total of about $1 \mathrm{fb}^{-1}$ collected during 2004 and 2005. The detection of a Lambda hyperon through its decay into a proton and a negative pion (with a branching ratio of $64 \%$ ) was used as tagging for events where a $\mathrm{K}^{-}$absorption occurs. The proton and pion tracks are identified with the DC and its momentum is measured with a precision better than $1 \%$ (in the case of the proton), and the decay vertex is known with an error below $1 \mathrm{~mm}$.

An additional proton that produces a vertex with the reconstructed lambda decay path is searched for, determining in this way the absorption vertex without the requirement of the identification of the interacting kaon track. The determination of the absorption vertex, will not only improve the resolution in the $\Lambda p$ invariant mass (through changing the quadrimomentum of the proton) but will also allow to determine more precisely the procedence of the interaction, since its radial position along the KLOE setup is known with a precision better than $2 \mathrm{~mm}$. For the selected $\Lambda p$ events, a check on the mass of the proton track can be done if it is associated with a hit in the electromagnetic calorimeter that surrounds the KLOE drift chamber. The mass calculated by time of flight is shown in figure 1.

The indentification of the absorption vertex inside the gas (active) volume of the KLOE drift chamber allows for an almost full acceptance for events with the described configuration. An acceptance function has been calculated using the Monte Carlo simulation of the process:

$K^{-}+{ }^{4} \mathrm{He} \rightarrow \Lambda+p+n+n$

where $K^{-}$is considered at rest, and the products are generated following the available phase space, covering the whole range of accepted momentum and angular correlations. Applying the same event selection described before for the real data we can calculate an acceptance function 
defined as:

$A=A\left(P_{\Lambda p}, \theta_{\Lambda p}, M_{\Lambda p}\right)$

where $P_{\Lambda p}$ is the added momentum for the Lambda and the proton, $\theta_{\Lambda p}$ is the angle between the two particles, and $M_{\Lambda p}$ is its invariant mass. The projection of the acceptance function in the invariant mass spectrum is shown in fig 2.
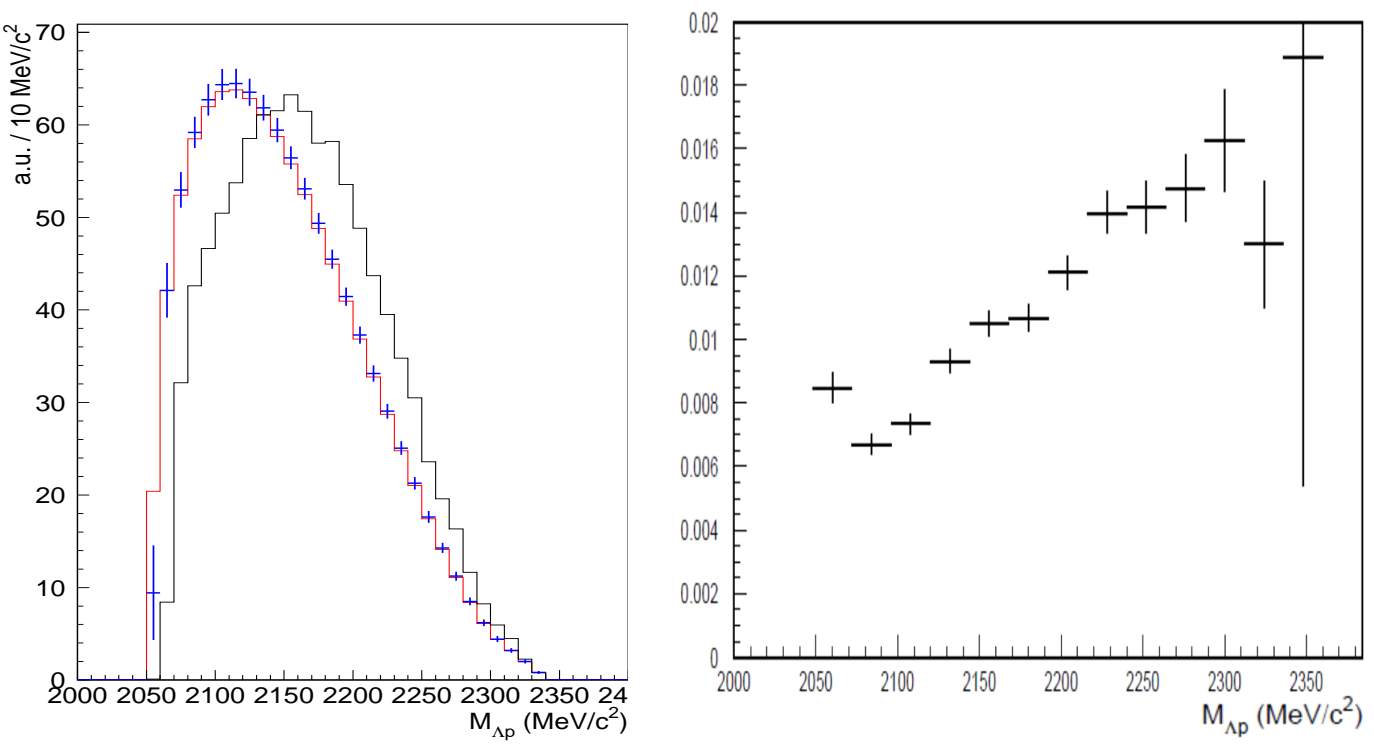

Figure 2: Left figure: Oiginally generated Monte Carlo invariant mass for $\Lambda p(r e d)$, and reconstructed output (black). The blue histogram shows the corrected reconstructed distribution after appying the acceptance correction. Normalization is arbitrary. Right figure: Acceptance correction projection into the $\Lambda p$ invariant mass. Normalization is arbitrary.

It can be notice how, even in the low invariant mass range (where the identification of low momentum protons is compromised), the acceptance does not present drop to zero. As expected, the acceptance for the high invariant mass range is not heavily distorted and the overall shape can be corrected for real events bin by bin with the calculated three dimensional acceptance function.

In the following section we see this correction applied to the $\Lambda p$ invariant mass distribution from the real data events.

\section{1 $\Lambda p$ invariant mass distribution and discussion.}

The final output of the $\Lambda p$ inclusive analysis can be seen in figure 3 , where the $\Lambda p$ invariant mass distribution is shown (it is rigorously speaking a $p p \pi^{-}$invariant mass distribution), in black before, and in red after the application of the acceptance correction. Please note that the corrected spectra has been arbitrarily normalized. A prominent enhacement is seen in the low invariant mass part of the spectrum.

This part of the spectrum is expected to be populated mainly by events coming from the single nucleon absorption process, where the kaon interacts with one nucleon producing an hyperon-pion pair, the proton in the final state coming from the initial nucleus (thus not participating in the interaction) or from the $\Sigma-\Lambda$ nuclear conversion process [10] in the residual nucleus. In the high 

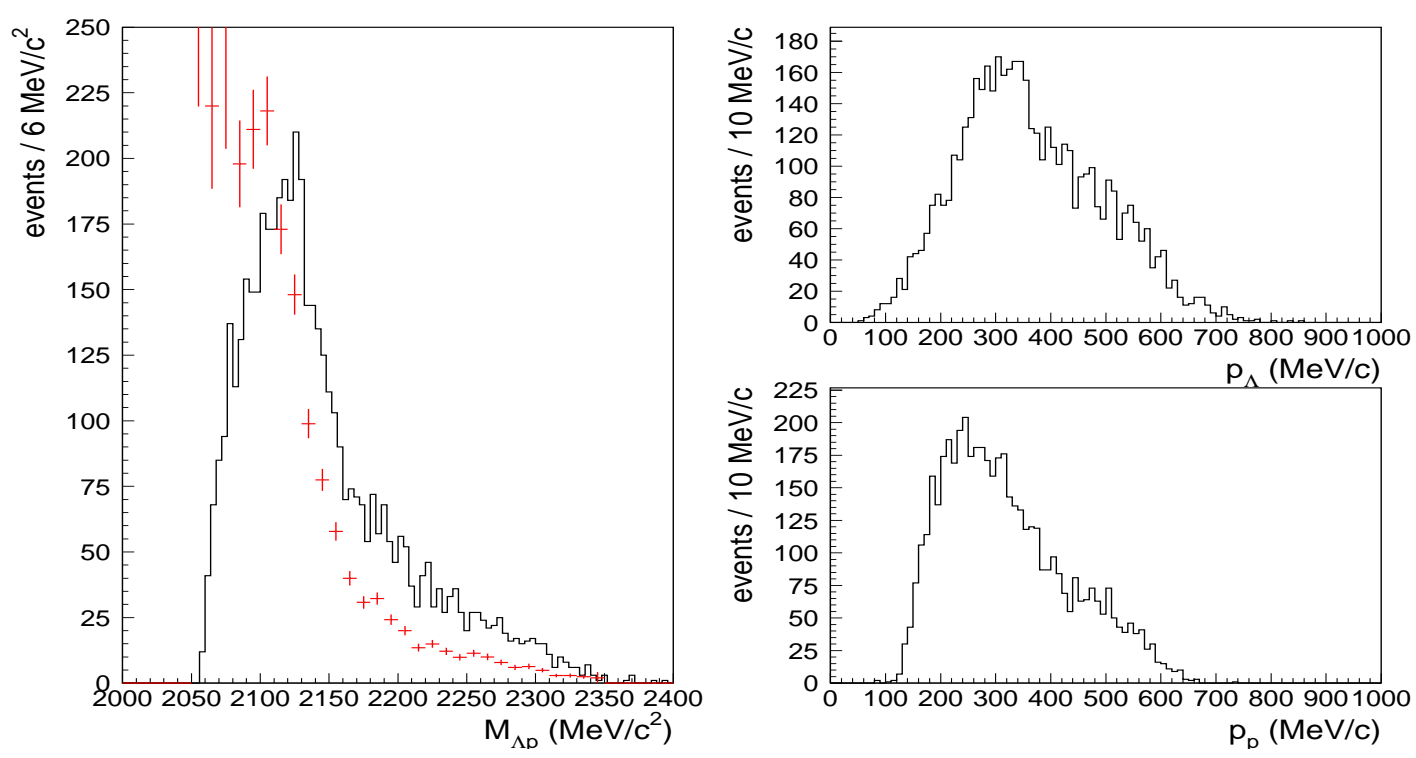

Figure 3: Invariant mass for $\Lambda p$ inclusive selection (left). In black the non-corrected spectrum, in red the acceptance corrected distribution (with arbitrary normalization). In the right part the momentum of the lambda (up) and proton (down) are shown.

invariant mass region (starting from $2150 \mathrm{MeV} / \mathrm{c}^{2}$ ) no clear structures are present. The momentum of the lambda and the proton is also shown in figure 3 .

As explained before, the knowledge of the shape of the $\Lambda p$ invariant mass for the single and multi-nucleon absorption processes is of great importance in the search for the kaonic bound clusters. The low invariant mass component in our spectrum, with predominant contribution from the mesonic channels, can in fact be explored in more detail. Following the same procedure used for the identification of the proton correlated with the absorption vertex, we search for a third particle, in this case a negative pion. This is indeed found in approximately $1 / 6$ of the $\Lambda p$ events, and the invariant mass and momentum distributions for this sub-sample are shown in figure 4 . With the requirement of the presence of a pion track, the contribution from the high momentum tail in the lambda and proton momentum loses strenght, if compared with the inclusive $\Lambda p$ selection.

We have performed a missing mass study in the hypothesis that the $\Lambda p$ pairs are generated by interactions of $K^{-}$at rest in ${ }^{4} \mathrm{He}$. The absorption in helium is expected to happen with 4 times more probability than in the carbon present in the isobutane, according to [11] (based on low energy $K^{-}$-nucleus scattering calculations [12][13]). For what concerns the contamination of inflight absorptions, a dedicated analysis of $\Lambda \pi^{-}$correlations in the KLOE data confirms that around $70 \%$ of the absorptions should be produced at rest. The missing mass spectra presented in figure 5 show a clear difference when comparing the inclusive $\Lambda p$ selection with the $\Lambda p \pi^{-}$exclusive events. This missing mass is defined as follows:

$$
M_{m i s s}^{2}=\left(p_{\text {init }}-p_{\Lambda}-p_{p}\right)^{2}
$$

where $p_{\text {init }}, p_{\Lambda}$ and $p_{p}$ are the 4-momenta of the inital state $K^{-}+{ }^{4} \mathrm{He}$ at rest, and the measured ones of lambda and proton, respectively. Since the missing mass represents the total energy of the undetected system in its center of mass frame, the events with $M_{\text {miss }}$ higher than $2 \times m_{N}+m_{\pi}(\sim$ 

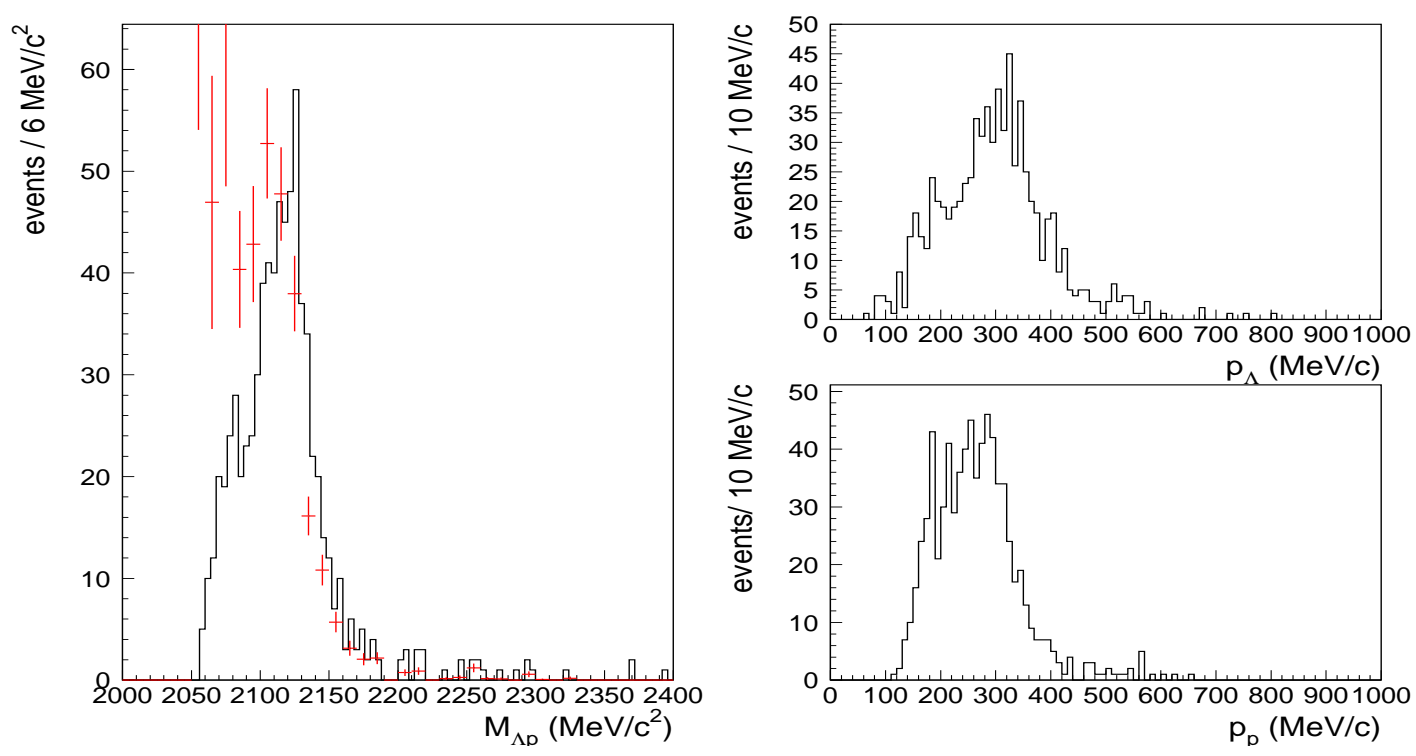

Figure 4: $\Lambda$ p invariant mass from the $\Lambda p \pi^{-}$events (left). In black the non-corrected spectrum, in red the acceptance corrected distribution (with arbitrary normalization). In the right part lambda (up) and proton (down) momentum distributions are shown.

$2015 \mathrm{MeV} / \mathrm{c}^{2}$ ) are considered to be dominated by the single nucleon absorption process, as it is indeed reflected by the data.

\section{Conclusions.}

The correlations of coincident $\Lambda p$ pairs produced in the interaction of $\mathrm{K}^{-}$coming from $\Phi$ decay in the gas volume of the KLOE Drift Chamber has been studied.

A detailed description of the invariant mass and momentum spectra for the inclusive $\Lambda p$ and exclusive $\Lambda p \pi^{-}$events has been presented, showing the later selection characteristics compatible with a predominant contribution from single nucleon absorption and $\Sigma$ - $\Lambda$ nuclear conversion processes.

The results show the acceptance for low momentum protons of the KLOE apparatus, allowing to detect low invariant mass $\Lambda p$ pairs, and proving how this detector is suited for performing lowenergy kaon-nucleon/nuclei interaction studies in a broad range of produced final states.

\section{References}

[1] Y. Akaishi and T. Yamazaki, Phys. Rev. C 65, 044005 (2002)

[2] M. Bazzi et al., Phys. Lett. B 704, 113-117 (2011).

[3] K. Suzuki et al., Nucl. Phys. A 827, 312C-314C (2012).

[4] G. Agakishiev et al., Phys. Lett. C 85, 035203 (2012).

[5] M. Agnello et al., Phys. Rev. Lett. 94, 919303 (2005). 

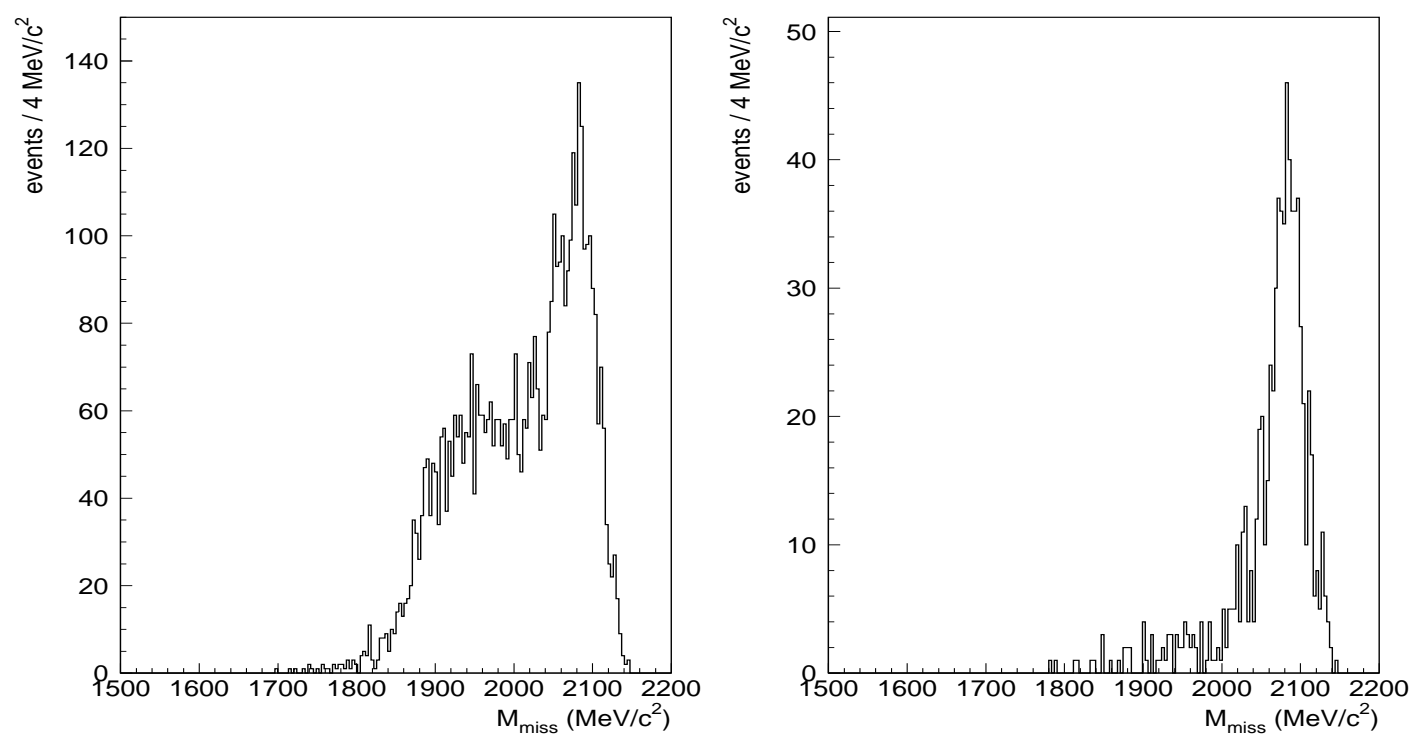

Figure 5: Missing mass (as defined in the text) for $\Lambda$ p inclusive selection (left) and $\Lambda p \pi^{-}$events (right).

[6] T. Suzuki et al., Mod. Phys. Lett. A 23, 2520-2523 (2008).

[7] P. A. Katz et al., Phys. Rev. D1 1267-1276 (1970).

[8] T. Buran et al., Phys. Lett. 20, 318 (1966).

[9] M. Adinolfi et al., Nucl. Instr. Meth. A 488, 51-73 (2002)

[10] C. Vander Velde-Wilquet et al., Nucl. Phys. A 241, 511 (1974).

[11] Kristian Piscicchia Doctoral thesis, Roma Tre University (2013).

[12] A. Deloff et al., Phys. Rev. C, 1688 (1974).

[13] C. Garcia-Recio et al., arXiv:nucl-th/0210030v2 (2003). 